\title{
Increased frequency of intermetatarsal and submetatarsal bursitis in early rheumatoid arthritis: a large case-controlled MRI study
}

Yousra J. Dakkak ${ }^{1 *}$ DD, Ellis Niemantsverdriet ${ }^{1}$, Annette H. M. van der Helm-van Mil ${ }^{1,2^{*}}$ and Monique Reijnierse ${ }^{3}$

\begin{abstract}
Background: The forefoot is a preferential location for joint and tendon sheath inflammation in rheumatoid arthritis (RA). It also contains bursae, of which the intermetatarsal bursae have a synovial lining. Some small imaging studies suggested that intermetatarsal bursitis (IMB) and submetatarsal bursitis (SMB) are involved in RA, but their association has not been thoroughly explored. Healthy control studies suggested that lesion size might be relevant. We studied the relation between IMB and SMB in early RA, compared to other arthritides and healthy controls, and the relevance of lesion sizes.
\end{abstract}

Methods: Six hundred and thirty-four participants were studied: 157 consecutive patients presenting with early RA, 284 other arthritides, and 193 healthy controls. All underwent unilateral contrast-enhanced MRI of the forefoot at presentation. Two readers independently scored IMB and SMB and measured transverse and dorsoplantar diameters, blinded to clinical data. Subsequently, consensus was reached. Intra-reader ICC was 0.89. Logistic regression models were used, and test characteristics were calculated.

Results: IMB and SMB associated with RA independent of each other $(P<0.001)$ and independent of age, gender, BMI, RA-MRI inflammation, and anti-CCP-antibodies $(P=0.041)$. Sensitivity for RA of IMB was $69 \%$, and for SMB $25 \%$. Specificity for IMB was 70\% compared to other arthritides, and 84\% compared to healthy controls. For SMB, this was $94 \%$ and $97 \%$ respectively. Regarding lesion size, the groups had considerable overlap: no cut-off size for RA could be distinguished with high sensitivity and specificity.

Conclusion: Intermetatarsal and submetatarsal bursitis associated with early rheumatoid arthritis, contributing to the emerging evidence that inflammation of juxta-articular soft tissues is an early feature of RA.

Keywords: Rheumatoid arthritis, Early arthritis, Healthy controls, Magnetic resonance imaging, Foot, Bursitis

\footnotetext{
*Correspondence: y.j.dakkak@lumc.nl; a.h.m.vanderhelm@erasmusmc.nl ${ }^{1}$ Department of Rheumatology, Leiden University Medical Center, P.O. Box 9600, 2300 RC Leiden, The Netherlands

Full list of author information is available at the end of the article
}

(C) The Author(s). 2020 Open Access This article is licensed under a Creative Commons Attribution 4.0 International License, which permits use, sharing, adaptation, distribution and reproduction in any medium or format, as long as you give appropriate credit to the original author(s) and the source, provide a link to the Creative Commons licence, and indicate if changes were made. The images or other third party material in this article are included in the article's Creative Commons licence, unless indicated otherwise in a credit line to the material. If material is not included in the article's Creative Commons licence and your intended use is not permitted by statutory regulation or exceeds the permitted use, you will need to obtain permission directly from the copyright holder. To view a copy of this licence, visit http://creativecommons.org/licenses/by/4.0/ The Creative Commons Public Domain Dedication waiver (http://creativecommons.org/publicdomain/zero/1.0/) applies to the data made available in this article, unless otherwise stated in a credit line to the data. 


\section{Key messages}

- Intermetatarsal bursitis and submetatarsal bursitis associated with RA, and intermetatarsal bursitis had the highest sensitivity (69\%).

- This contributes to the emerging evidence that in early RA extra-articular synovial inflammation is common.

- Regarding lesion size no cut-off for disease with high sensitivity and specificity could be distinguished.

\section{Background}

Rheumatoid arthritis (RA) is a systemic autoimmune disease characterized by chronic, persistent inflammation of the synovium-lined joints and tendon sheaths $[1,2]$. Preferential locations are the small joints of hands and feet. It has become apparent that early treatment with disease modifying anti-rheumatic drugs (DMARDs) improves disease outcome of RA patients [3]. Since prompt treatment has limited radiographic detectable damage, the European League Against Rheumatism (EULAR) recommends MRI for the early detection and follow-up of RA as it sensitively measures inflammation. According to the RA-MRI score (RAMRIS), inflammation is defined as synovitis, tenosynovitis, and bone marrow edema/osteitis $[4,5]$. In the forefoot, however, imaging also reveals the presence and/or inflammation of bursae. So far, bursitis in the forefeet has gained little attention in RA literature.

Interestingly, naturally present bursae possess a synovial lining similar to synovial joints and tendon sheaths [6]. In the forefoot, intermetatarsal bursae are present, anatomically without a connection to the metatarsophalangeal (MTP) joints [7]. Two ultrasound studies have reported intermetatarsal bursitis (IMB) in patients with established RA $[8,9]$. Additionally, one MRI study in 70 asymptomatic volunteers reported on fluid in the intermetatarsal spaces as a sign of IMB [10]. Gadolinium contrast enhancement was not used as a measure of inflammation. The authors suggested that a transverse diameter of $\leq 3 \mathrm{~mm}$ could be considered physiologic; however, these results have not been validated [10]. Thus, while data suggest that IMB may be associated with RA, its involvement in early disease has not been studied systematically.

In the subcutaneous fat, submetatarsal bursae that lack a synovial lining may develop due to high pressure and friction, leading to collagen degradation and localized fluid-collection that is visible on imaging as a sharply demarcated area in the submetatarsal space [11]. An ultrasound study reported submetatarsal bursitis (SMB) in patients with established RA [12]. However, this has also been observed in healthy controls. To the best of our knowledge, regarding IMB and SMB, no direct comparison has been made between RA and other arthritides, which is a comparison that is clinically relevant [13]. Also, no comparison has been made between RA and healthy controls, to analyze whether these findings could be normal.

Additionally, in the forefoot, Morton's neuroma (MN) and diffuse submetatarsal alterations (DSMA) in the subcutis have been described. MN may emerge due to mechanical irritation, or secondary to IMB, and has been described in RA as well as asymptomatic volunteers [14-16]. DSMA consist of fibrosis or inflammatory tissue that may be induced by mechanical stress and may represent early stages in the development of SMB, particularly under the first and fifth metatarsal heads [17].

Overall, the current available literature does not give a fulfilling and thorough picture of the prevalence of IMB and SMB in RA patients. Therefore, this large crosssectional MRI study was set up to study (1) the association of these lesions in RA, compared to other arthritides and to healthy controls, and (2) whether the size of these lesions can differentiate between diseases and healthy controls. Although not the main focus of this study, MN and DSMA were studied in subanalyses.

\section{Materials and methods Participants}

Between June 2013 and March 2016, 447 consecutive patients newly presenting with clinical arthritis of a symptom duration $<2$ years who were naïve to DMAR Ds were included in the Leiden Early Arthritis Cohort (EAC) [18]. The Leiden University Medical Center is the only rheumatology referral center within the Leiden area. Inclusion in the EAC of consecutive early arthritis patients has been part of regular care since 1993 [19]. Contrast-enhanced MRI of the forefoot was added to the protocol in June 2013. At baseline, swollen joint counts were performed, serum samples were obtained, and patients underwent MRI. Six MRI-examinations were excluded because of inhomogeneous fat suppression, 441 examinations remained (flowchart in Supplementary Figure S1). RA was defined as a clinical diagnosis of RA plus fulfillment of the 2010 RA criteria during the first year of follow-up [1]. The remaining patients received alternative diagnoses and were grouped together as 'other arthritides' (Table 1).

Healthy controls were recruited by advertisements in local newspapers and websites, as reported previously [20]. Participants had no history of inflammatory rheumatic disease, no joint symptoms during the last month, and no arthritis at physical examination.

The EAC and healthy control study were approved by the local medical ethics committee (approval numbers P10.108 and P17.261). Informed consent was obtained. 
Table 1 Baseline characteristics of all participants

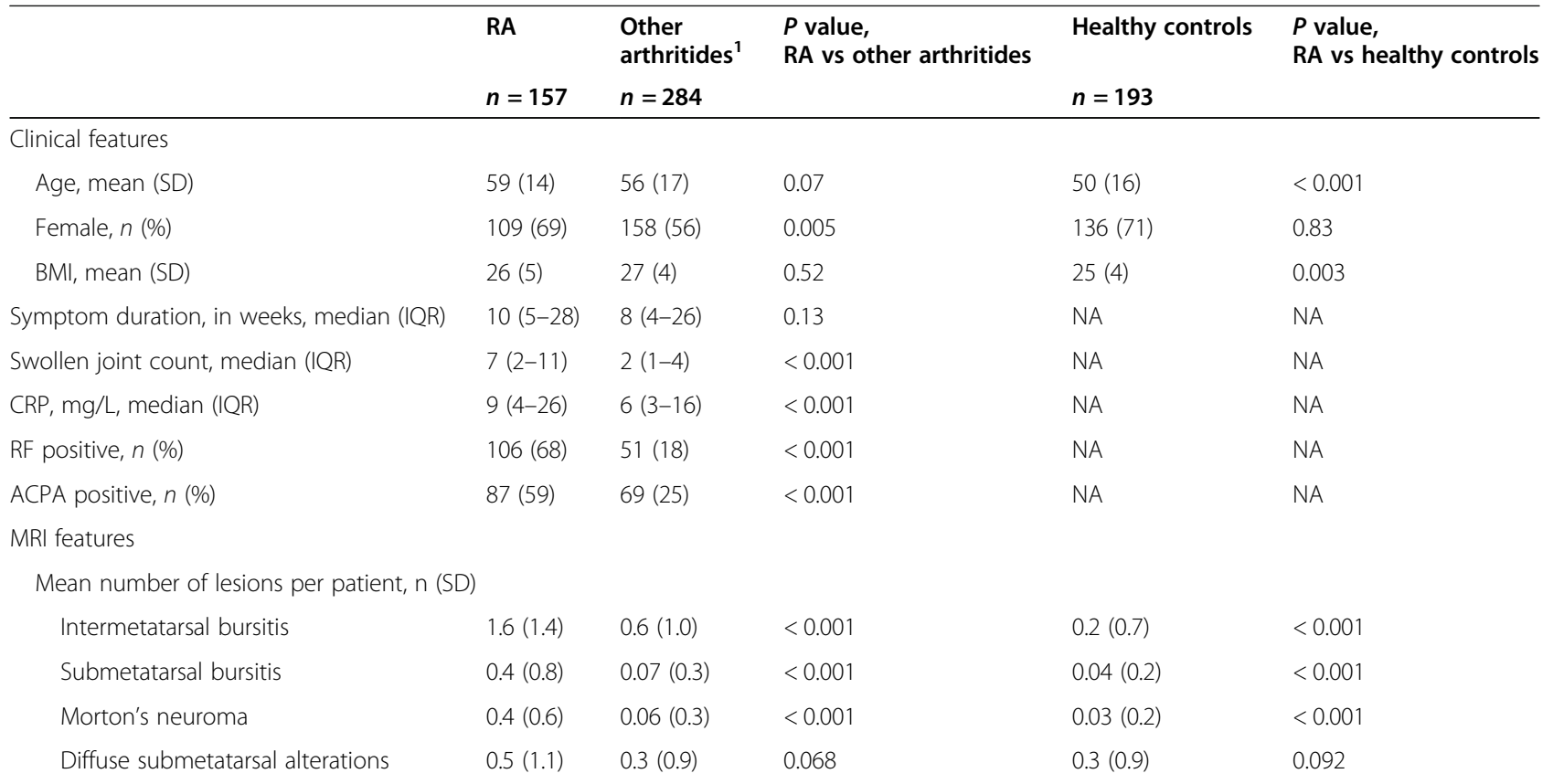

$R A$ rheumatoid arthritis, $S D$ standard deviation, $B M I$ body mass index, IQR interquartile range, $C R P$ C-reactive protein, $R F$ rheumatoid factor, $A C P A$ anti-citrullinated peptide antigen (anti-CCP), MRI magnetic resonance imaging, NA not applicable

${ }^{1}$ This group included the following diagnoses: unclassified arthritis $(n=148)$, psoriatic arthritis or spondyloarthritis $(n=45)$, inflammatory osteoarthritis $(n=23)$, reactive arthritis $(n=7)$, crystal arthropathy $(n=21)$, remitting seronegative symmetrical synovitis with pitting edema ( $n=12)$, and other diagnoses $(n=28)$

\section{MRI scanning}

Patients and symptom-free persons were scanned according to our routine MRI protocol as described in Supplementary Data S1. They underwent unilateral MRI of the hand and forefoot of the more painful side, or the dominant side in case of symmetrical symptoms, $\leq 2$ weeks after the first presentation and before start of DMARDs. In symptom-free persons, the dominant side was scanned. A musculoskeletal extremity 1.5-T MRI unit (Oni; GE Healthcare, Madison, WI, USA) was used with a $145-\mathrm{mm}$ coil. Acquired sequences for the forefoot after intravenous injection of gadolinium contrast included: axial T1weighted fast spin-echo with fat suppression (repetition time ms/echo time ms 700/9.5; acquisition matrix $364 \times 224$, echo train length 2) and coronal T1-weighted fast spin-echo with fat suppression (540/ 7.5 ; acquisition matrix $320 \times 192$, echo train length 2 ) . Field-of-view was $140 \mathrm{~mm}$, slice thickness $3 \mathrm{~mm}$, and slice gap $0.3 \mathrm{~mm}$ for both planes. Axial sequences had 14 slices, and coronal sequences 20 slices.

\section{MRI evaluation}

\section{Anatomy and scoring system}

The forefoot was divided into four intermetatarsal and five submetatarsal spaces (Fig. 1) [21]. The intermetatarsal space is dorsally bound by the deep dorsal aponeurosis and plantar by the superficial transverse metatarsal ligament [6, 7]. It is divided into a superior and inferior level by the deep transverse metatarsal ligament. The intermetatarsal bursae lie in the superior intermetatarsal spaces [6], the neurovascular bundle, from which $\mathrm{MN}$ originates, lies in the inferior intermetatarsal space and has a close cohesion with the synovial lining of the intermetatarsal bursae $[6,7]$. The submetatarsal spaces lie in the subcutis, plantar to the superficial transverse metatarsal ligament, and extend until the epidermis. We used the following definitions:

- Intermetatarsal bursitis (IMB). Contrast enhancement in the superior intermetatarsal space with or without rim enhancement

- Submetatarsal bursitis (SMB). A sharply demarcated area with contrast enhancement in the submetatarsal space with or without rim enhancement

- Morton's neuroma (MN). An isolated spindleshaped lesion in the inferior intermetatarsal space with or without contrast enhancement, without rim enhancement continuous with IMB [10]

- Diffuse submetatarsal alterations (DSMA). An unsharply defined area in the submetatarsal space with diffuse contrast enhancement

Lesions had to be visible on two consecutive slices in both axial and coronal planes. Besides scoring for 


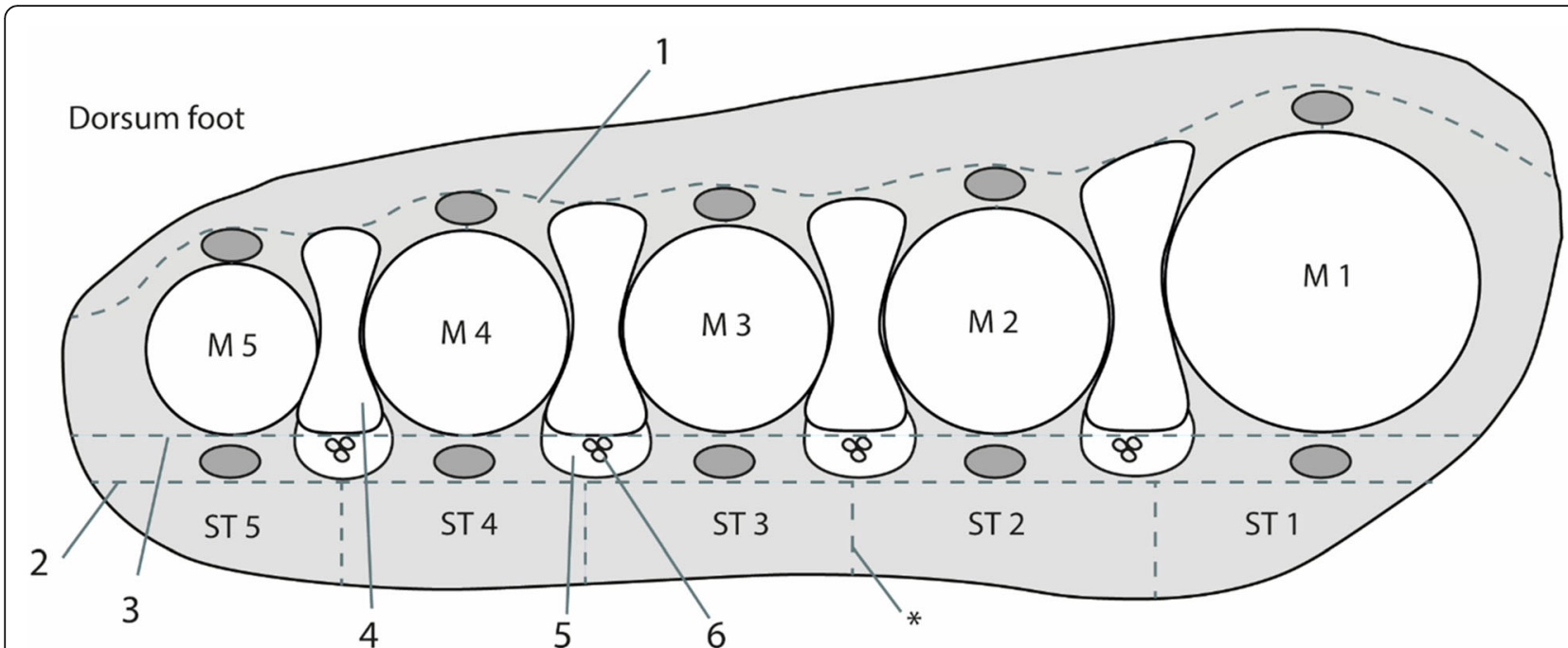

Fig. 1 Schematic illustration of the forefoot at the metatarsal heads with intermetatarsal and submetatarsal spaces. The intermetatarsal space is demarcated at the dorsal side by the deep dorsal aponeurosis (1) and at the plantar side by the superficial transverse metatarsal ligament (2). The deep transverse metatarsal ligament (3) divides the intermetatarsal space into a superior (4) and inferior part (5), respectively, containing the bursa and neurovascular bundle (6). The submetatarsal spaces (ST) are located in the subcutis and are artificially bordered by the midline of the intermetatarsal space ${ }^{*}$ ). M, metatarsal heads. Gray ovals represent extensor and flexor tendons of the forefoot

presence, the transverse and dorsoplantar diameter of $\mathrm{IMB}, \mathrm{SMB}$, and $\mathrm{MN}$ were measured in millimeters on coronal images. DSMA was not measured as, per definition, it has no sharply demarcated borders.

\section{Scoring}

Two raters (a musculoskeletal radiologist with 23 years of experience, and a trained MD with 2 years of experience in RAMRIS scoring and has scored $>400$ MRIs according to this system during a training period of several months prior to evaluating the MRIs that are part of this study) $[22,23]$, who were blinded to all clinical data, independently scored the MRIs. The two raters determined the final scores by consensus: a lesion was only regarded to be present if both readers agreed on this. First, all early arthritis patients were scored, followed by healthy controls. To exclude observer bias introduced by knowing that images belonged to healthy controls, MRIs of healthy controls were randomly mixed with 20 MRIs from EAC patients. Based upon these 20 patients that were scored twice an intra-reader intraclass correlation coefficients (ICC) was determined that was 0.89 .

In addition, to explore whether intermetatarsal and submetatarsal lesions were associated with RA independent of RAMRIS inflammation, the MRIs were also scored for synovitis, tenosynovitis, and osteitis at MTP joints in line with the RAMRIS by two independent readers, also blinded to clinical data, as is described in Supplementary Data S1 [22-25]. RAMRIS inflammation was considered present when synovitis, tenosynovitis, and/or osteitis were scored as $\geq 1$ by both readers.

\section{Analyses}

IMB and SMB were studied in RA patients, other arthritides, and healthy controls. First, as patients could have more than one lesion, e.g., one patient could have IMB at more than one location, the mean number of lesions in RA patients was compared to patients with other arthritides, and to healthy controls using MannWhitney $U$ tests.

Next, the data was dichotomized for presence per lesion: e.g., whether a patient had IMB at any location. Logistic regression analysis was used to compare RA to other arthritides and to healthy controls. Multivariable models corrected for the simultaneous occurrence of the different lesions and for age, gender, BMI, presence of RAMRIS inflammation, and anti-citrullinated peptide antibodies (anti-CCP), as these may be important in the relation between these lesions and RA [26, 27]. Test characteristics for RA were determined. Heatmaps were plotted with the percentage of participants with a lesion per location. Finally, measured transverse and dorsoplantar diameters were plotted to assess whether a cutoff for disease could be observed.

As subanalyses the analyses were repeated for $\mathrm{MN}$ and DSMA.

Calculations were performed with SPSS Statistics, version 23.0; IBM, Armonk, NY. $P<0.05$ was considered statistically significant. 


\section{Results}

\section{Patient characteristics}

Of the 441 consecutively included EAC patients, 157 were classified as early RA, 284 patients had other arthritides (Table 1). One hundred and ninety-three healthy controls were recruited. RA patients were predominantly female (69\%) and had a median symptom duration of 10 weeks (interquartile range: 5-28).

RA patients had a higher number of IMB and SMB lesions per patient compared to other arthritides and to healthy controls (all $P<0.001$ ) (Table 1 ). Next, the presence of a lesion was dichotomized as described in the Methods.

\section{Association of inter- and submetatarsal bursitis with RA}

The number of participants with lesions is given in Table 2. RA patients more often had IMB and SMB than other arthritides (all $P<0.001$ ).

Since IMB and SMB were both associated with RA, and in addition age, gender, BMI, RAMRIS inflammation, and anti-CCP-antibodies may be important in the relation between the lesions and RA, a multivariable model was performed that included both lesions and these clinical parameters [26, 27]. IMB and SMB remained associated with RA independent of these factors (Table 2); the effect size was largest for IMB (OR $4.5,95 \%$ CI 2.7-7.8).

The analyses were repeated comparing RA patients to healthy controls, revealing similar results (Supplementary Table S1).

\section{Test characteristics of intermetatarsal and submetatarsal} bursitis for RA

Next, test characteristics were determined for IMB and SMB (Supplementary Table S2). Sensitivity for IMB was $69 \%$, and for SMB 25\%. Specificity of IMB compared to other arthritides was 70\%, and compared to healthy controls $84 \%$. For SMB, this was $94 \%$ and $97 \%$, respectively.
Heatmap of lesions for RA, other arthritides, and healthy controls

The distribution of the lesions was plotted in heatmaps (Fig. 2, Supplementary Table S3). In RA, IMB affected the 3rd intermetatarsal space most (57\% of patients). Regarding SMB, the first and fifth submetatarsal space were most affected: in $13 \%$ and $12 \%$ of patients, respectively.

Similarly, in other arthritides and healthy participants, the 3rd intermetatarsal space was affected by IMB most often, but with lower frequency (20\% and $11 \%$ respectively). For SMB at the first and fifth submetatarsal space this was $2 \%$ and $4 \%$, respectively, in other arthritides and $1 \%$ and $2 \%$ in healthy controls. MRI-examples are given in Fig. 3.

\section{Transverse and dorsoplantar diameter of lesions for} patients with rheumatoid arthritis, other arthritides, and healthy controls

Next, the transverse and dorsoplantar diameters of IMB and $\mathrm{SMB}$ were measured (in $\mathrm{mm}$ ) and plotted in histograms (Fig. 4). We evaluated cut-offs based upon the histograms (Supplementary Table S4).

First the transverse diameter of IMB was studied. From the histograms it was apparent that lesions $\geq 4$ $\mathrm{mm}$ were infrequent in other arthritides (3\% of patients) and healthy controls (2\%). We studied whether this could be used as a cut-off for RA: however lesions $\geq 4$ $\mathrm{mm}$ were also less frequent in RA (13\%). In all groups the majority of lesions were $\leq 3 \mathrm{~mm}$ : $68 \%$ of RA patients had a lesion $\leq 3 \mathrm{~mm}$, compared to $29 \%$ other arthritides and $15 \%$ of healthy controls. Hence, a cut-off of $3 \mathrm{~mm}$ increased specificity to $97 \%$ compared to other arthritides and $98 \%$ to healthy controls, at the cost of a decrease in sensitivity to $13 \%$.

Theoretically, IMB may be limited by the metatarsal heads in its ability to distend transversely and may, therefore, upon inflammation, distend dorsoplantar. A dorsoplantar diameter of $\mathrm{IMB} \geq 15 \mathrm{~mm}$ was infrequent in the control groups (Fig. 4). Taking $\geq 15 \mathrm{~mm}$ as a cut-

Table 2 The association of intermetatarsal and submetatarsal lesions with early RA compared to other early arthritides

\begin{tabular}{|c|c|c|c|c|c|c|c|c|}
\hline & \multicolumn{2}{|c|}{ Participants with MRI features, $n$ (\%) } & \multicolumn{2}{|c|}{ Univariable analyses } & \multicolumn{2}{|l|}{$\begin{array}{l}\text { Multivariable } \\
\text { analysis }^{1}\end{array}$} & \multicolumn{2}{|l|}{$\begin{array}{l}\text { Multivariable } \\
\text { analyses }^{2}\end{array}$} \\
\hline & RA & Other arthritides & $O R(95 \% \mathrm{Cl})$ & $P$ value & OR $(95 \% \mathrm{Cl})$ & $P$ value & OR $(95 \% \mathrm{Cl})$ & $P$ value \\
\hline Intermetatarsal bursitis & $109(69)$ & $84(30)$ & $5.4(3.5-8.3)$ & $<0.001$ & $4.5(2.7-7.8)$ & $<0.001$ & $3.7(2.1-6.6)$ & $<0.001$ \\
\hline Submetatarsal bursitis & $39(25)$ & $17(6)$ & $5.2(2.8-9.5)$ & $<0.001$ & $2.2(1.03-4.5)$ & 0.041 & $2.3(1.1-4.8)$ & 0.031 \\
\hline Morton's neuroma & $30(19)$ & $10(4)$ & $6.7(3.2-14.2)$ & $<0.001$ & - & - & $3.1(1.3-7.7)$ & 0.012 \\
\hline Diffuse submetatarsal alterations & $36(23)$ & $45(16)$ & $1.6(0.9-2.6)$ & 0.067 & - & - & $0.9(0.5-1.8)$ & 0.86 \\
\hline
\end{tabular}

The results of logistic regression analyses are presented. $R A$ rheumatoid arthritis, $O R$ odds ratio, $C l$ confidence interval

${ }^{1}$ Multivariable model including intermetatarsal bursitis, submetatarsal bursitis, age, gender, anti-CCP, and RAMRIS inflammation (defined as the presence of synovitis, tenosynovitis, and/or osteitis)

${ }^{2}$ Multivariable model including intermetatarsal bursitis, submetatarsal bursitis, Morton's neuroma, diffuse submetatarsal alterations, age, gender, BMI, anti-CCP antibodies, and RAMRIS inflammation 

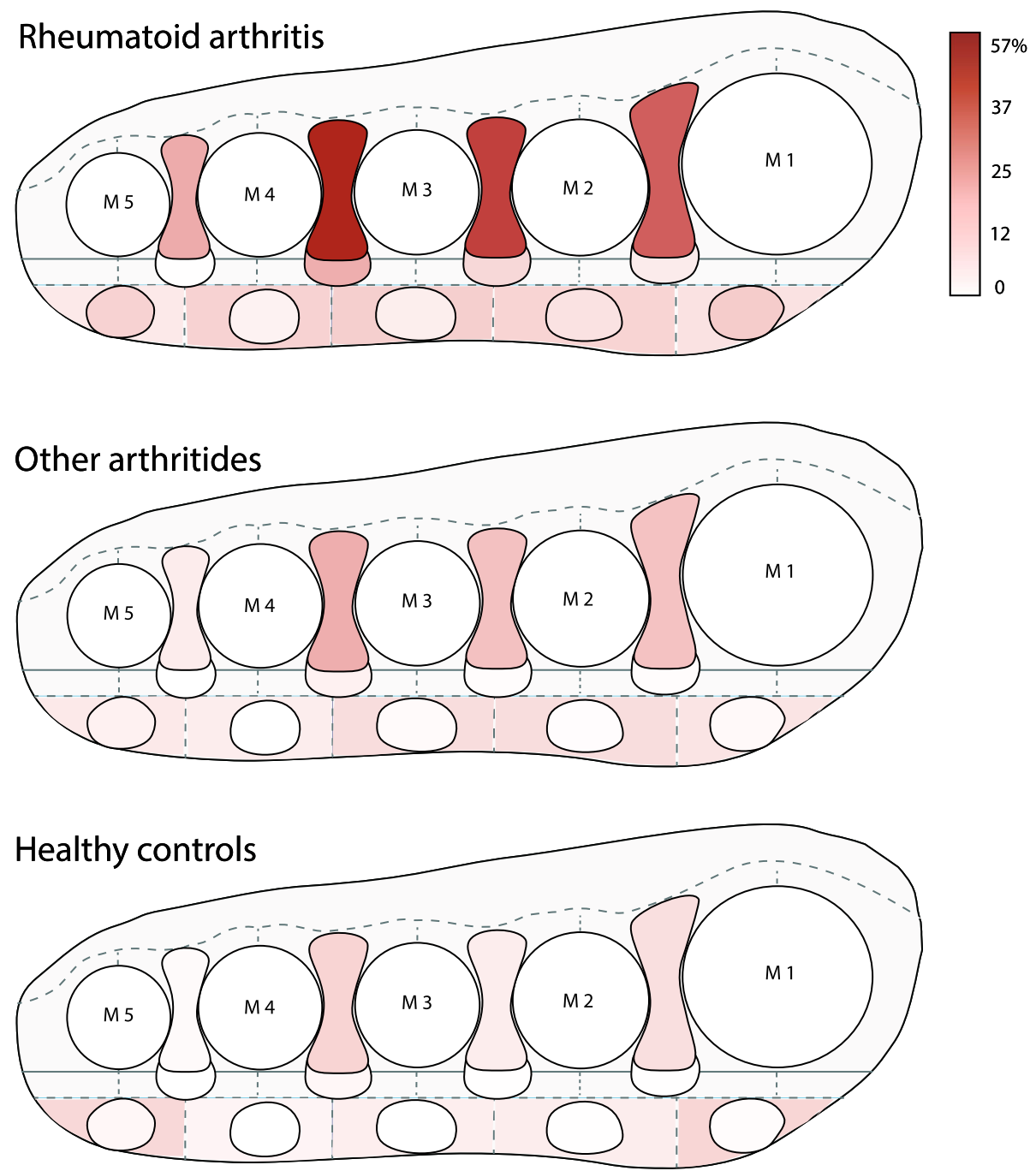

Fig. 2 Heatmap of intermetatarsal bursitis, submetatarsal bursitis, Morton's neuroma, and diffuse submetatarsal alterations for every population. Schematic illustration in coronal view of the frequency of lesions in each compartment of the forefoot at the level of the metatarsal heads (see also supplementary Table S3). The frequency of the lesions (\% of participants in the respective group) is represented by an increase in color intensity. The compartments are defined in Fig. 1. Mortons neuroma (MN) is demarcated plantar to intermetatarsal bursitis (IMB). In the subcutis, submetatarsal bursitis (SMB) is illustrated as a demarcated oval. The remainder of the subcutis represents diffuse submetatarsal alterations (DSMA). $I M B, S M B$, and MN are most frequently seen in RA. The second and third IMB are preferred locations whereas the fourth is the least involved. In the subcutis of RA patients, DSMA is seen under MTP 2, 3, and 4, whereas in healthy controls, this is seen under MTP 1 and 5. SMB dominates under MTP 1 and 5 in RA patients. M, metatarsal heads

off increased specificity for RA compared to other arthritides (95\%) and healthy controls (100\%). Sensitivity, however, decreased to $16 \%$.

Similarly, regarding SMB no cut-off could be determined that did not lead to a substantial decrease in sensitivity for RA (Supplementary Table S4).

\section{Subanalyses for MN and DSMA}

MN was associated with RA compared to other arthritides in univariable and multivariable analyses that included IMB, SMB, MN, DSMA and clinical parameters $(P<0.001$ and $P=0.012$ respectively). DSMA was not associated ( $P=0.069$ and $P=0.86$, respectively) (Table $2)$. Similar results were obtained when compared to healthy controls (Supplementary Table S1).

The test characteristics for MN were determined and were as follows: sensitivity $19 \%$, and specificity $96 \%$ compared to other arthritides and 98\% compared to healthy controls. For DSMA this was not determined as it was not associated with RA.

MN and DSMA were incorporated in the heatmaps in Fig. 2 (Supplementary Table S3 presents the exact frequency per location). MN occurred most frequently in the third intermetatarsal space (20\% of RA patients). 

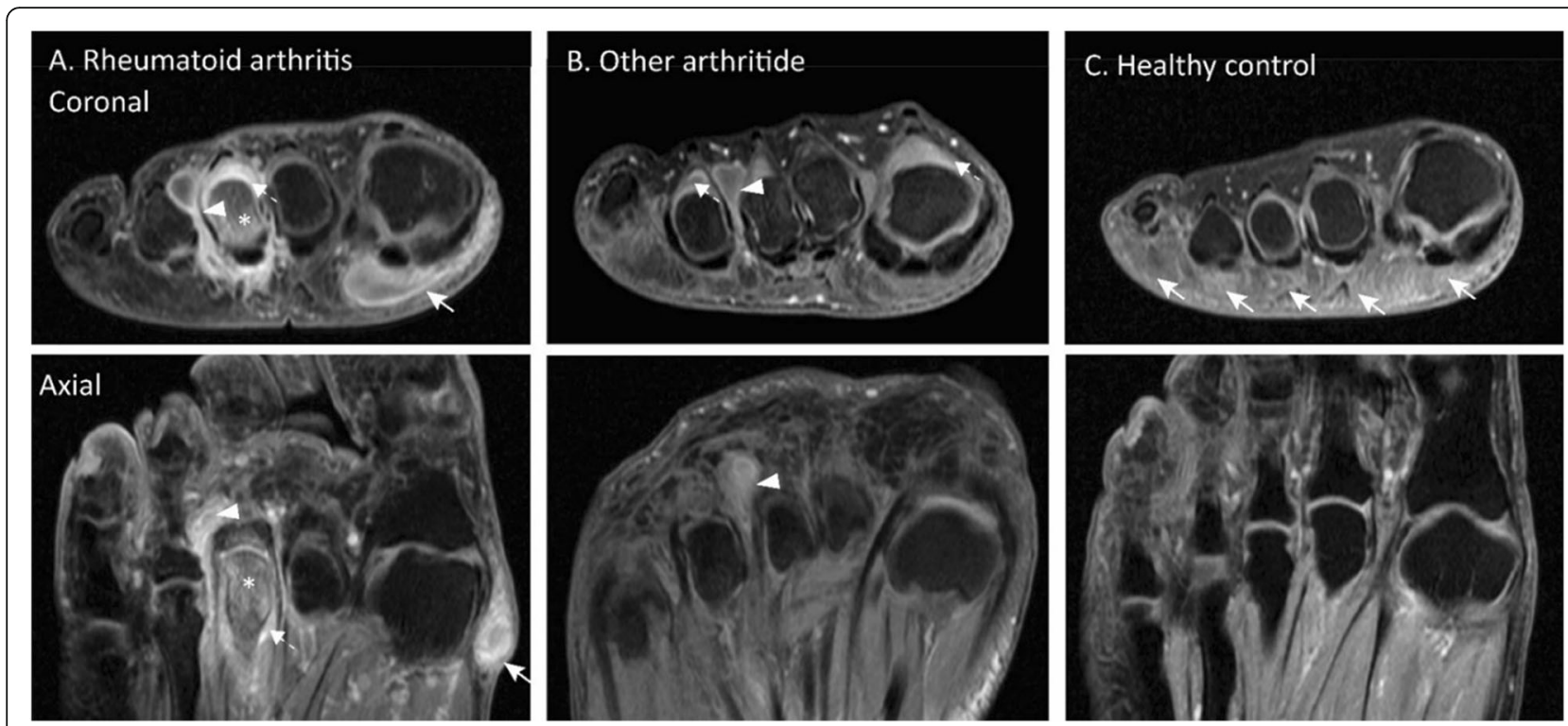

Fig. 3 MR examples of intermetatarsal and submetatarsal bursae. Coronal and axial fat suppressed T1-weighted FSE gadolinium-enhanced images of the forefoot at the level of the metatarsal heads. a Female participant with RA (age 61 years) with intermetatarsal (IMB) and submetatarsal bursitis (SMB). IMB in the 3rd intermetatarsal space (arrowhead) with peripheral enhancement protruding dorsal (dumbbell shape) and plantar (teardrop shape) of the metatarsal heads. Peripheral enhancement of a mass in the first submetatarsal space, consistent with SMB (white arrow). Synovitis of MTP 3 (dotted arrow), as well as osteitis in the head of the third metacarpal bone and proximal phalanx $(*)$ ). b Female participant with another arthritide (diagnosis of viral reactive arthritis, age 34 years) and IMB at the 3rd intermetatarsal space (arrowhead) with dorsal protrusion. Additional synovitis of MTP 1 and 4 (dotted arrows). c Female healthy control (age 50 years) with diffuse submetatarsal alterations (DSMA) in all submetatarsal spaces, predominantly visible at the 1st, 2nd, and 4th submetatarsal spaces (arrows). Intense linear contrast enhancement at the 2nd intermetatarsal space is consistent with a small vessel on the consecutive slices (not shown), there is no IMB visible on the axial images

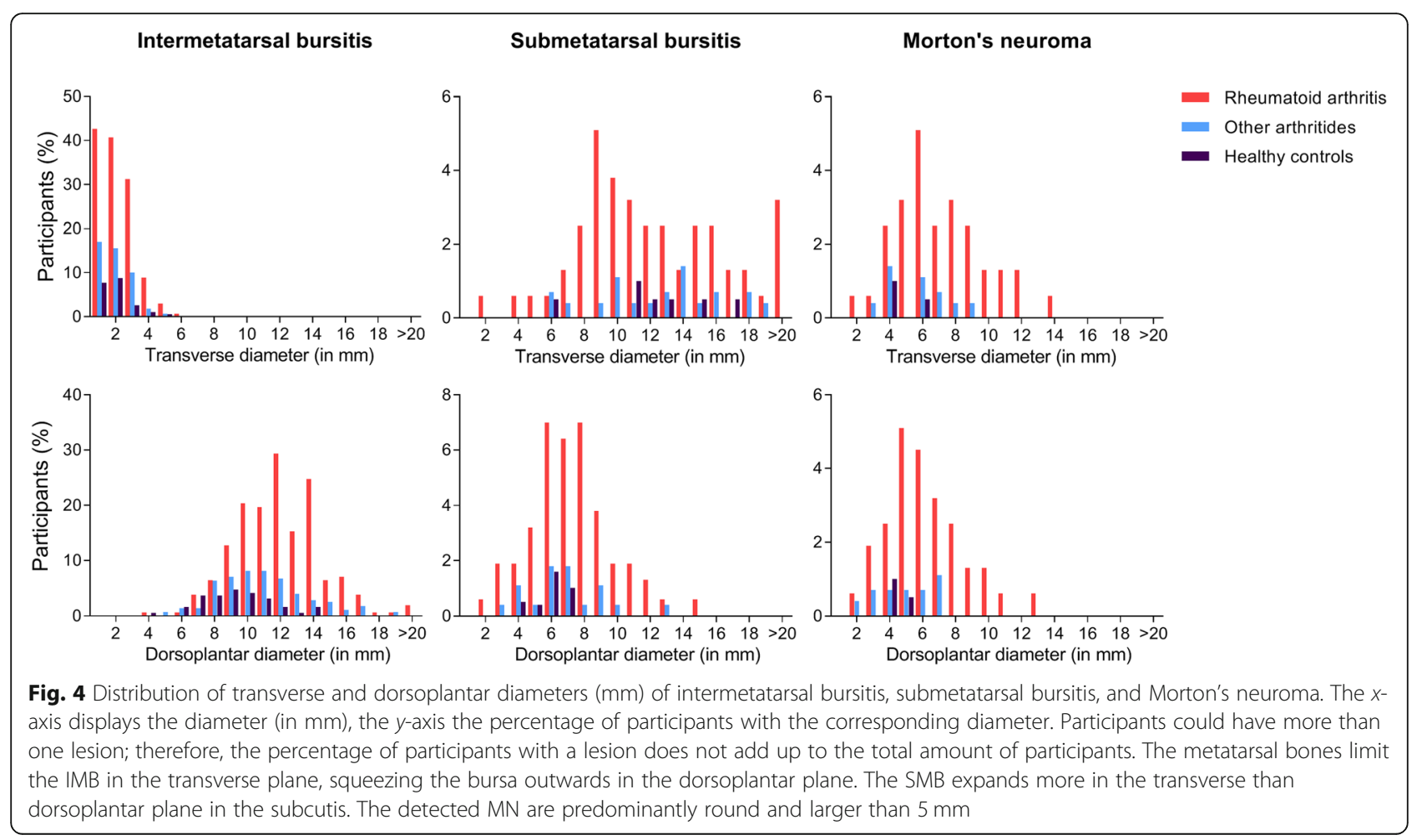


DSMA in the first and fifth submetatarsal space were relatively more common in healthy controls (both locations $10 \%)$.

Regarding lesion size for $\mathrm{MN}$, a transverse diameter of $\geq 7 \mathrm{~mm}$ was infrequent in control groups (Fig. 4): using this cut-off increased specificity for RA, however sensitivity decreased to $12 \%$. Additionally, literature suggests a cut-off of $\geq 5 \mathrm{~mm}$, this decreased sensitivity to $16 \%$ [10, 28]. Similarly, for the dorsoplantar diameter, using a $\geq 4 \mathrm{~mm}$ cut-off based on Fig. 4 decreased sensitivity to 13\% (Supplementary Table S4). DSMA was not measured as, per definition, it has no sharply demarcated borders.

\section{Discussion}

This cross-sectional study aimed to explore MRIdetected IMB and SMB in consecutive patients presenting with early RA, compared to other arthritides and healthy controls. We observed that both IMB and SMB were associated with and specific for RA (specificity ranging from 70 to $97 \%$ ). In addition, we studied whether lesion size might be relevant and found considerable overlap in size between the groups; therefore, no cut-off for RA could be distinguished with high sensitivity and specificity. To our knowledge, our study is the largest to date to systematically study involvement of bursae in the forefoot in RA.

IMB results from inflammation of a naturally present, synovium-lined, structure [6]. Since RA is a disease of the synovium, the bursae might be a primary focus of disease, previously unnoticed. It can be hypothesized that IMB occurs secondary to concomitant arthritis; however, there is no anatomical connection between intermetatarsal bursae and MTP joints and in our study IMB associated with RA independent of the presence of RAMRIS inflammation, defined as synovitis, tenosynovitis, and osteitis [7].

In the plantar subcutaneous fat of the forefoot SMB was scored as sharply demarcated submetatarsal areas of contrast enhancement with or without rim enhancement that may develop secondary to mechanical loading [17]. These non-native (adventitious) bursae form at sites of friction but have imaging features similar to native bursae [29].

In addition MN and DSMA were scored, of which MN was also associated with RA. This association remained present after correcting for age, gender, and BMI; features that may relate to other pathologies predisposing to MN. Thus, MN seems to be increased in RA. Within RA, IMB has previously been suggested as a cause of MN [14, 30]. Inflamed bursae may irritate the common plantar digital nerve $[6,30]$, with secondary $\mathrm{MN}$ formation $[7,30]$. This may explain why MN mostly occurred at locations where IMB was most frequent (second and third intermetatarsal spaces). For the definition of $\mathrm{MN}$, besides the shape and location, it was important in our study that the lesion did not have any rim enhancement that was continuous with the IMB. This might be especially challenging at the second and third intermetatarsal spaces, where enlarged intermetatarsal bursae can extend below the deep transverse metatarsal ligament towards the neurovascular bundle [10]. In the literature the prevalence for $\mathrm{MN}$ varies between studies, from 15\% in symptomatic volunteers to $54 \%$ in asymptomatic volunteers $[10,16,31]$. An explanation for the low frequency of $20 \%$ in our study could be the challenging differentiation between $\mathrm{MN}$ and IMB and potential coexistence of the two, especially for smaller MN. The limited MRI protocol, without T1 and fluid-sensitive sequences, may underscore fibrous MN [32]. Therefore, no definitive conclusion regarding the frequency of $\mathrm{MN}$ can be drawn from our study. We do not believe this undermines the association that was found of MN with RA, as the protocol restriction applies for all study groups.

DSMA, interestingly, occurred in healthy controls specifically under the first and fifth metatarsal heads. These locations are consistent with a previous study and are considered a normal finding based on mechanical loading [17]. In RA, DSMA occurred predominantly under MTP 2, 3, and 4, whereas under MTP 1 and 5 SMB were seen (Fig. 2).

It is difficult to compare our findings to previous studies in RA, as they utilized ultrasound and included patients with established RA receiving treatment rather than early disease $[8,33]$.

The intermetatarsal space is limited in the transverse plane by the metatarsal bones. Thus, bursitis is squeezed either dorsal and or plantar, especially at the second and third intermetatarsal spaces, respectively called a dumbbell or a teardrop phenomenon (Fig. 1), explaining the larger dorsoplantar diameter that is found (Fig. 4) [7, 21].

Our study has some limitations. First, the scoring method is not validated; therefore, reading was done by consensus rather than by independent readers, as this may be necessary in the setting of preliminary findings [34]. However, for determining the validity of an outcome measure, it is crucial to demonstrate the reliability of scoring between independent readers [34, 35]. The Intra-reader ICC, however, was determined and was reassuring. Second, our MRI protocol contained no fluidsensitive sequences, as the protocol was originally intended to score RAMRIS inflammation. Therefore, small amounts of fluid in the bursae may have remained undetected, leading to an underestimation of observed lesions. Nevertheless, the use of contrast enhancement might be a strength, which previous studies in early arthritis found essential for optimal assessment of MRIdetected synovitis and tenosynovitis [25, 36]. Because 
intermetatarsal bursae have a synovial lining, we assumed that contrast enhancement would increase sensitivity of synovial hypertrophy in (active) bursitis. Thus, although theoretically small amounts of fluid in a bursa may be missed without a fluid-sensitive sequence, this could be normal in a bursa and rarely occurs at this location without enhancing synovial hypertrophy [10, 37]. The greater prevalence of contrast-enhancing (teno)synovitis might be expected to occur in RA. To account for this we included RAMRIS inflammation in our multivariable model. Third, although the lesions were associated with RA independent of the factors that were adjusted for, additional factors might be of influence to the occurrence of these lesions, such as physical activity and type of shoes that were unaccounted for and may potentially be a source of bias. Also, we did not include a comparison with weight-bearing radiographs $[38,39]$. Finally, our study was cross-sectional in nature. Thus, although our study suggests that these lesions may aid the clinician as a (differential) diagnostic tool, prospective studies are warranted to further establish the diagnostic relevance of these lesions.

\section{Conclusion}

IMB and SMB are both associated with RA. As IMB has a synovial lining, these results contribute to the emerging evidence that in early RA, besides intra-articular synovitis, juxta-articular synovial inflammation is common. Previously, tenosynovitis was reported [18, 36], and now IMB as well. The current findings pose questions on whether the bursitis correlated to symptoms, responds to therapy, or has diagnostic value, e.g., in predicting RA development in the early phases of disease.

\section{Supplementary Information}

Supplementary information accompanies this paper at https://doi.org/10. 1186/s13075-020-02359-w.

Additional file 1.

\section{Abbreviations}

RA: Rheumatoid arthritis; IMB: Intermetatarsal bursitis; SMB: Submetatarsal bursitis; MRI: Magnetic resonance imaging; DMARDs: Disease modifying antirheumatic drugs; EULAR: European League Against Rheumatism; RAMRIS: RAMRI score; MTP joint: Metatarsophalangeal joint; MN: Morton's neuroma; DSMA: Diffuse submetatarsal alterations; EAC: Early Arthritis Cohort; ICC: Intraclass correlation coefficients; anti-CCP: Anti-citrullinated peptide antibodies

\section{Acknowledgements}

We would like to thank the patients and healthy controls who took the time to participate.

\section{Authors' contributions}

All authors contributed to the conception and study design. YJD analyzed the data. YJD, EN, AHMvdHvM, and MR contributed to acquisition and interpretation of the data. YJD and MR wrote the first version of the manuscript and all authors revised it critically. All authors read and approved the final version of the document.

\section{Funding}

This work was supported by the European Research Council (ERC) under the European Union's Horizon 2020 research and innovation program (starting grant, agreement no. 714312) and the Dutch Arthritis Foundation.

The funding source had no role in the design and conduct of the study; collection, management, analysis, and interpretation of the data; preparation, review, or approval of the manuscript; or decision to submit the manuscript for publication.

\section{Availability of data and materials \\ The datasets analyzed during the current study are available from the corresponding author on reasonable request.}

\section{Ethics approval and consent to participate}

The performed study complies with the Declaration of Helsinki, and the locally appointed ethics committee has approved the early arthritis cohort and healthy control studies (approval numbers P10.108 and P17.261, respectively) and informed consent has been obtained from the subjects.

\section{Consent for publication}

Not applicable.

\section{Competing interests}

None declared.

\section{Author details}

${ }^{1}$ Department of Rheumatology, Leiden University Medical Center, P.O. Box 9600, 2300 RC Leiden, The Netherlands. 'Department of Rheumatology, Erasmus Medical Center, Rotterdam, The Netherlands. ${ }^{3}$ Department of Radiology, Leiden University Medical Center, Leiden, The Netherlands.

Received: 10 September 2020 Accepted: 19 October 2020

Published online: 23 November 2020

\section{References}

1. Aletaha D, Neogi T, Silman AJ, Funovits J, Felson DT, Bingham CO 3rd, et al. 2010 rheumatoid arthritis classification criteria: an American College of Rheumatology/European League Against Rheumatism collaborative initiative. Arthritis Rheum. 2010;62(9):2569-81.

2. Rogier $C$, Hayer S, van der Helm-van Mil A. Not only synovitis but also tenosynovitis needs to be considered: why it is time to update textbook images of rheumatoid arthritis. Ann Rheum Dis. 2020;79:546-7.

3. Smolen JS, Landewe R, Breedveld FC, Buch M, Burmester G, Dougados M, et al. EULAR recommendations for the management of rheumatoid arthritis with synthetic and biological disease-modifying antirheumatic drugs: 2013 update. Ann Rheum Dis. 2014;73(3):492-509.

4. Ostergaard M, Bird P, Gandjbakhch F, Eshed I, Haugen IK, Haavardsholm EA et al. The OMERACT MRI in Arthritis Working Group - update on status and future research priorities. J Rheumatol. 2015:42(12):2470-2.

5. Colebatch AN, Edwards CJ, Ostergaard M, van der Heijde D, Balint PV, D'Agostino MA, et al. EULAR recommendations for the use of imaging of the joints in the clinical management of rheumatoid arthritis. Ann Rheum Dis. 2013;72(6):804-14

6. Chauveaux D, Le Huec JC, Midy D. The supra-transverse intermetatarsocapital bursa: a description and its relation to painful syndromes of the forefoot. Surg Radiol Anat. 1987;9(1):13-8.

7. Theumann NH, Pfirrmann CW, Chung CB, Mohana-Borges AV, Haghighi P, Trudell DJ, et al. Intermetatarsal spaces: analysis with MR bursography, anatomic correlation, and histopathology in cadavers. Radiology. 2001; 221(2):478-84

8. Bowen CJ, Culliford D, Dewbury K, Sampson M, Burridge J, Hooper L, et al. The clinical importance of ultrasound detectable forefoot bursae in rheumatoid arthritis. Rheumatology (Oxford). 2010;49(1):191-2.

9. Hammer HB, Kvien TK, Terslev L. Intermetatarsal bursitis is frequent in patients with established rheumatoid arthritis and is associated with anti-cyclic citrullinated peptide and rheumatoid factor. RMD Open. 2019:5(2):e001076.

10. Zanetti M, Strehle JK, Zollinger H, Hodler J. Morton neuroma and fluid in the intermetatarsal bursae on MR images of 70 asymptomatic volunteers. Radiology. 1997;203(2):516-20. 
11. Van Hul E, Vanhoenacker F, Van Dyck P, De Schepper A, Parizel PM. Pseudotumoural soft tissue lesions of the foot and ankle: a pictorial review. Insights Into Imaging. 2011;2(4):439-52.

12. Bowen CJ, Hooper L, Culliford D, Dewbury K, Sampson M, Burridge J, et al. Assessment of the natural history of forefoot bursae using ultrasonography in patients with rheumatoid arthritis: a twelve-month investigation. Arthritis Care Res (Hoboken). 2010;62(12):1756-62.

13. Schunemann HJ, Oxman AD, Brozek J, Glasziou P, Bossuyt P, Chang S, et al. GRADE: assessing the quality of evidence for diagnostic recommendations. Evid Based Med. 2008;13(6):162-3.

14. Awerbuch MS, Shephard E, Vernon-Roberts B. Morton's metatarsalgia due to intermetatarsophalangeal bursitis as an early manifestation of rheumatoid arthritis. Clin Orthop Relat Res. 1982;167:214-21.

15. Erickson SJ, Canale PB, Carrera GF, Johnson JE, Shereff MJ, Gould JS, et al. Interdigital (Morton) neuroma: high-resolution MR imaging with a solenoid coil. Radiology. 1991;181(3):833-6

16. Symeonidis PD, Iselin LD, Simmons N, Fowler S, Dracopoulos G, Stavrou P. Prevalence of interdigital nerve enlargements in an asymptomatic population. Foot Ankle Int. 2012;33(7):543-7.

17. Studler U, Mengiardi B, Bode B, Schottle PB, Pfirrmann CW, Hodler J, et al. Fibrosis and adventitious bursae in plantar fat pad of forefoot: MR imaging findings in asymptomatic volunteers and MR imaging-histologic comparison. Radiology. 2008;246(3):863-70.

18. Dakkak YJ, Jansen FP, DeRuiter MC, Reijnierse M, van der Helm-van Mil AHM. Rheumatoid Arthritis and Tenosynovitis at the Metatarsophalangeal Joints: An Anatomic and MRI Study of the Forefoot Tendon Sheaths. Radiology. 2020;295(1):146-54. https://doi.org/10.1148/radiol.2020191725. Epub 2020 Feb 11

19. de Rooy DP, van der Linden MP, Knevel R, Huizinga TW, van der Helm-van Mil AH. Predicting arthritis outcomes--what can be learned from the Leiden Early Arthritis Clinic? Rheumatology (Oxford). 2011;50(1):93-100.

20. Mangnus L, van Steenbergen HW, Reijnierse M, van der Helm-van Mil AH Magnetic resonance imaging-detected features of inflammation and erosions in symptom-free persons from the general population. Arthritis Rheumatol. 2016;68(11):2593-602.

21. Cherry L, King L, Thomas M, Roemer F, Culliford D, Bowen CJ, et al. The reliability of a novel magnetic resonance imaging-based tool for the evaluation of forefoot bursae in patients with rheumatoid arthritis: the FFB score. Rheumatology (Oxford). 2014;53(11):2014-7.

22. Ostergaard M, Peterfy C, Conaghan $\mathrm{P}$, McQueen F, Bird P, Ejbjerg B, et al. OMERACT rheumatoid arthritis magnetic resonance imaging studies. Core set of MRI acquisitions, joint pathology definitions, and the OMERACT RAMRI scoring system. J Rheumatol. 2003;30(6):1385-6.

23. Haavardsholm EA, Ostergaard M, Ejbjerg BJ, Kvan NP, Kvien TK. Introduction of a novel magnetic resonance imaging tenosynovitis score for rheumatoid arthritis: reliability in a multireader longitudinal study. Ann Rheum Dis. 2007; 66(9):1216-20.

24. Dakkak YJ, Matthijssen XME, van der Heijde D, Reijnierse M, van der Helmvan Mil AHM. Reliability of Magnetic Resonance Imaging (MRI) Scoring of the Metatarsophalangeal Joints of the Foot according to the Rheumatoid Arthritis MRI Score. J Rheumatol. 2020;47(8):1165-73. https://doi.org/10.3899/ jrheum.190258.

25. Stomp W, Krabben A, van der Heijde D, Huizinga TW, Bloem JL, van der Helm-van Mil AH, et al. Aiming for a shorter rheumatoid arthritis MRI protocol: can contrast-enhanced MRI replace T2 for the detection of bone marrow oedema? Eur Radiol. 2014;24(10):2614-22.

26. Nieuwenhuis WP, Mangnus L, van Steenbergen HW, Newsum EC, Huizinga TW, Reijnierse $M$, et al. Older age is associated with more MRI-detected inflammation in hand and foot joints. Rheumatology (Oxford). 2016;55(12):2212-9.

27. Mangnus L, Nieuwenhuis WP, van Steenbergen HW, Huizinga TW, Reijnierse M, van der Helm-van Mil AH. Body mass index and extent of MRI-detected inflammation: opposite effects in rheumatoid arthritis versus other arthritides and asymptomatic persons. Arthritis Res Ther. 2016;18(1):245.

28. Redd RA, Peters VJ, Emery SF, Branch HM, Rifkin MD. Morton neuroma: sonographic evaluation. Radiology. 1989;171(2):415-7.

29. Ruangchaijatuporn T, Gaetke-Udager K, Jacobson JA, Yablon CM, Morag Y. Ultrasound evaluation of bursae: anatomy and pathological appearances. Skelet Radiol. 2017:46(4):445-62.

30. Bossley CJ, Cairney PC. The intermetatarsophalangeal bursa--its significance in Morton's metatarsalgia. J Bone Joint Surg Br. 1980;62-b(2):184-7.
31. lagnocco A, Coari G, Palombi G, Valesini G. Sonography in the study of metatarsalgia. J Rheumatol. 2001;28(6):1338-40.

32. Zanetti M, Weishaupt D. MR imaging of the forefoot: Morton neuroma and differential diagnoses. Semin Musculoskelet Radiol. 2005:9(3):175-86.

33. Hooper L, Bowen CJ, Gates L, Culliford D, Arden NK, Edwards CJ. Comparative distribution of ultrasound-detectable forefoot bursae in patients with osteoarthritis and rheumatoid arthritis. Arthritis Care Res. 2014; 66(6):869-77.

34. Bankier AA, Levine D, Halpern EF, Kressel HY. Consensus interpretation in imaging research: is there a better way? Radiology. 2010;257(1):14-7.

35. Boers M, Kirwan J, Tugwell P, Beaton D, Bingham CO 3rd, Conaghan $P$, et al. The OMERACT handbook; 2017 (Updated December-20-17). p. 1-111.

36. Ostergaard M, Conaghan PG, O'Connor P, Szkudlarek M, Klarlund M, Emery $P$, et al. Reducing invasiveness, duration, and cost of magnetic resonance imaging in rheumatoid arthritis by omitting intravenous contrast injection -does it change the assessment of inflammatory and destructive joint changes by the OMERACT RAMRIS? J Rheumatol. 2009;36(8):1806-10.

37. Stomp W, Krabben A, van der Heijde D, Huizinga TW, Bloem JL, Ostergaard $\mathrm{M}$, et al. Aiming for a simpler early arthritis MRI protocol: can Gd contrast administration be eliminated? Eur Radiol. 2015:25(5):1520-7.

38. Fuhrmann RA, Layher F, Wetzel WD. Radiographic changes in forefoot geometry with weightbearing. Foot Ankle Int. 2003:24(4):326-31.

39. Stomp W, Krabben A, van der Helm-van Mil A, Reijnierse M. Effects of wearing high heels on the forefoot: an MRI evaluation. Scand J Rheumatol. 2014;43(1):80-1.

\section{Publisher's Note}

Springer Nature remains neutral with regard to jurisdictional claims in published maps and institutional affiliations.
Ready to submit your research? Choose BMC and benefit from:

- fast, convenient online submission

- thorough peer review by experienced researchers in your field

- rapid publication on acceptance

- support for research data, including large and complex data types

- gold Open Access which fosters wider collaboration and increased citations

- maximum visibility for your research: over $100 \mathrm{M}$ website views per year

At $\mathrm{BMC}$, research is always in progress.

Learn more biomedcentral.com/submissions 